\title{
Abundant progenitor cells in the adventitia contribute to atherosclerosis of vein grafts in ApoE-deficient mice
}

\author{
Yanhua Hu, ${ }^{1}$ Zhongyi Zhang,, Evelyn Torsney, ${ }^{1}$ Ali R. Afzal, ${ }^{1}$ Fergus Davison, ${ }^{1}$ \\ Bernhard Metzler, ${ }^{2}$ and Qingbo $\mathbf{X u}^{1}$ \\ 1Department of Cardiac and Vascular Sciences, St George's Hospital Medical School, London, United Kingdom. 2Division of Cardiology, \\ Department of Internal Medicine, University Hospital, Innsbruck, Austria.
}

\begin{abstract}
Recent evidence indicates that vascular progenitor cells may be the source of smooth muscle cells (SMCs) that accumulate in atherosclerotic lesions, but the origin of these progenitor cells is unknown. To explore the possibility of vascular progenitor cells existing in adults, a variety of tissues from ApoE-deficient mice were extensively examined. Immunohistochemical staining revealed that the adventitia in aortic roots harbored large numbers of cells having stem cell markers, e.g., Sca-1 $1^{+}(21 \%)$, c-kit ${ }^{+}(9 \%), \mathrm{CD}^{+} 4^{+}(15 \%)$, and Flk $1^{+}$cells (4\%), but not SSEA-1 ${ }^{+}$embryonic stem cells. Explanted cultures of adventitial tissues using stem cell medium displayed a heterogeneous outgrowth, for example, islands of round-shaped cells surrounded by fibroblast-like cell monolayers. Isolated Sca-1 ${ }^{+}$cells were able to differentiate into SMCs in response to PDGF-BB stimulation in vitro. When Sca- $1^{+}$cells carrying the LacZ gene were transferred to the adventitial side of vein grafts in ApoE-deficient mice, $\beta-\mathrm{gal}^{+}$cells were found in atherosclerotic lesions of the intima, and these cells enhanced the development of the lesions. Thus, a large population of vascular progenitor cells existing in the adventitia can differentiate into SMCs that contribute to atherosclerosis. Our findings indicate that ex vivo expansion of these progenitor cells may have implications for cellular, genetic, and tissue engineering approaches to vascular disease.
\end{abstract}

\section{Introduction}

It is believed that smooth muscle cells (SMCs) in atherosclerosis are derived from the media of the artery in response to PDGF released by injured endothelial cells and aggregated platelets (1). However, this concept is challenged by recent findings demonstrating that other sources of SMCs may contribute to vascular diseases (2-8). It is also evidenced that SMCs in atherosclerotic lesions differ from those in the media $(9,10)$. We observed that new SMCs in vein grafts appear in the neointima earlier than in the media after massive cell death, which is an early cellular event in the grafted vessels (11). Furthermore, a recent study demonstrated that smooth muscle progenitors were present in circulating blood (12), although their origins are unknown. Concomitantly, we showed that about $60 \%$ of SMCs in atherosclerotic lesions of vein grafts were derived from the donor vessel wall and $40 \%$ from recipients, possibly from circulating blood $(8,13)$. These findings strongly suggest the possibility of stem or progenitor cells being the source of smooth muscle accumulation in atherosclerotic lesions.

The question is whether SMCs within atherosclerotic lesions are derived from bone marrow cells. Based on double staining for $\alpha$-actin and GFP, Sata et al. (7) suggested that bone marrow cells can differentiate into SMCs to participate in the formation of neointimal and atherosclerotic lesions, but our data derived from SM22-LacZ mice expressing the LacZ gene only in SMCs did not support the ability of bone marrow cells to differentiate

Nonstandard abbreviations used: smooth muscle (SM); smooth muscle cell (SMC); stage-specific embryonic antigen-1 (SSEA-1); stem cell antigen-1 (Sca-1). Conflict of interest: The authors have declared that no conflict of interest exists.

Citation for this article: J. Clin. Invest. 113:1258-1265 (2004).

doi:10.1172/JCI200419628. into SMCs in lesions (8). After these controversial results were discussed in the correspondence section of Circulation (14), Tanaka et al. reported that bone marrow cells cannot differentiate into mature SMCs within neointimal lesions of moderately injured vessels (15). Thus, additional sources of the SMCs that form atherosclerotic lesions could exist, which led us to search further for these progenitor cells.

The vascular adventitia is defined as the outermost connective tissue of vessels. Recently, the adventitia was increasingly considered a highly active segment of vascular tissue that contributes to a variety of disease pathologies, including atherosclerosis and restenosis (16-20). For instance, Shi et al. (21) showed that in carotid artery-vein grafts, neointimal proliferation is preceded by activation and proliferation of adventitial fibroblasts, which are differentiated into myofibroblasts and migrate to the neointima. Adventitial cells can also produce reactive oxygen species via activation of NADPH oxidase, which may play a more extensive role in the control of vascular tone (19). However, no data exist concerning the presence of stem or progenitor cells in the adventitia. Using ApoE knockout mice combined with vein graft models $(22,23)$, the present study was designed to identify the presence of stem cells in the vessel wall and to clarify whether these cells could participate in the lesion formation in vein grafts. We demonstrated that progenitor cells were abundant in the adventitia and could differentiate into SMCs in vitro and in vivo.

\section{Results}

Progenitor cells in the adventitia. To search additional sources of progenitor cells, we examined almost all types of tissues in ApoEdeficient mice by immunostaining. Cells expressing progenitor cell markers in non-bone marrow tissues, e.g., brain, muscle, liver, 

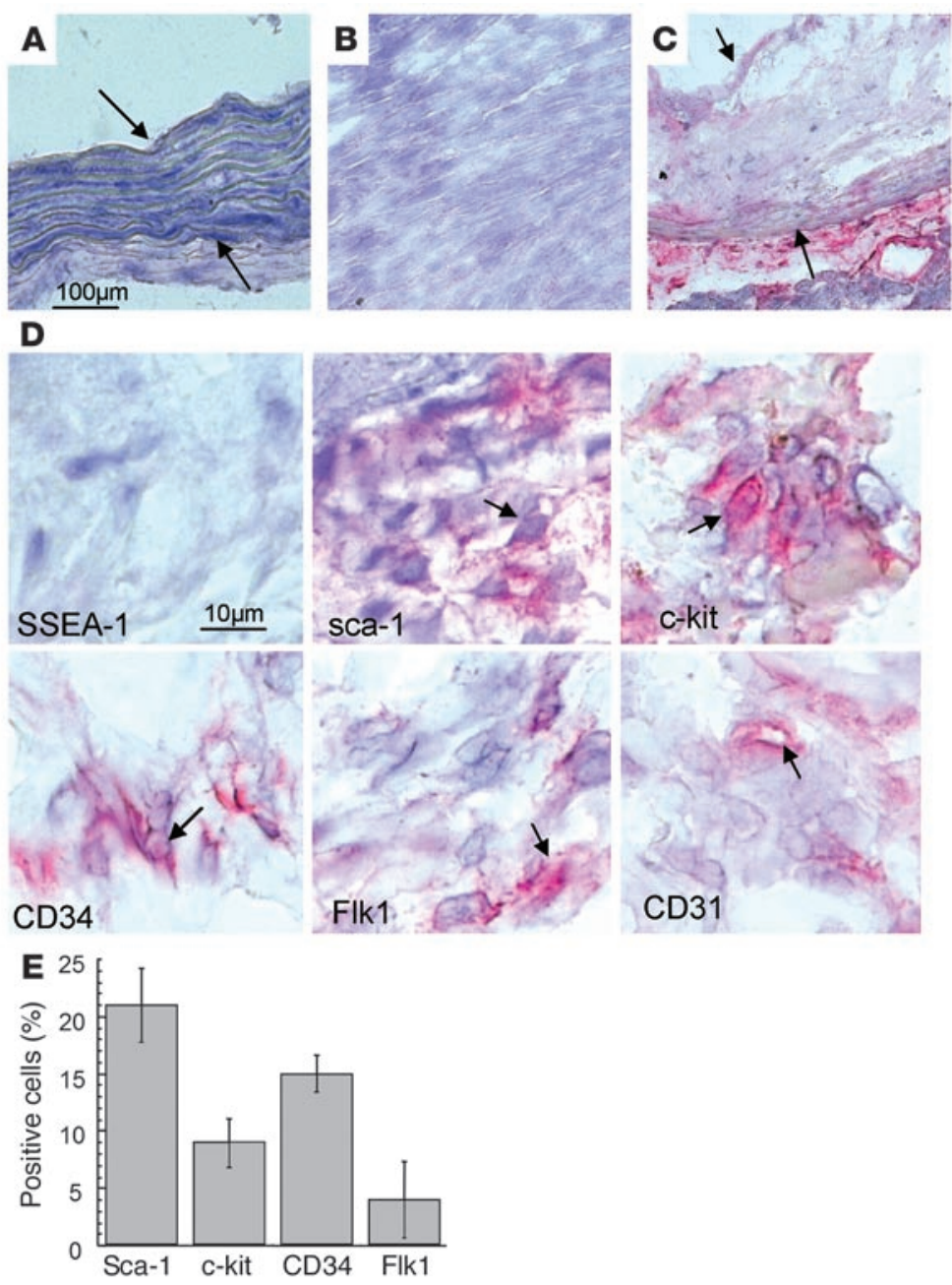

heart, kidney, spleen, and lung, were labeled with selected antibodies to the markers, i.e., stage-specific embryonic antigen-1 (SSEA-1), stem cell antigen-1 (Sca-1), c-kit, CD133, CD34, and Flk1. Within the organs examined, less than $0.01 \%$ of cells were found to be positive (data not shown). Surprisingly, we found that only the adventitia of the vessel wall contained large numbers of these marker-positive cells (Figure 1), and it was rare to see positive cells in the intima and media of the artery (Figure 1A) and heart (Figure 1B) tissues. No positive staining for the embryonic stem cell marker SSEA-1 was found, whereas $21 \%$ and $9 \%$ of total cells in the adventitia of the aortic root showed either Sca-1 or c-kit positivity (Figure 1, C, D, and E). CD $34^{+}$and Flk $1^{+}$cells were also found in the adventitia in ratios of $13 \%$ and $4 \%$, respectively (Figure 1, D and $\mathrm{E}$ ). These positive cells were more abundant in the region of the aortic root than in other areas of the adventitia. Staining for Sca-1, c-kit, CD34, and Flk1 was not found in the aortic intima and media (Figure 1A for Sca-1; other data not shown). In addition, $\mathrm{CD} 133^{+}$cells in the adventitia were less than $0.1 \%$, indicating the difference in progenitor cell composition between the adventitia and bone marrow tissues.

Because Sca- $1^{+}$cells were abundant, adventitial cells from aortic roots were cultivated using stem cell culture medium to prevent cell differentiation. Data shown in Figure 2 indicate the heterogeneous structure of cultured adventitial cells, including fibroblast-

\section{Figure 1}

Immunohistochemical staining. Aortic roots from $\mathrm{ApoE}^{-/-}$ mice were sectioned and labeled with a variety of antibodies as indicated. Sections were developed with alkaline phosphatase anti-alkaline phosphatase (APAAP) techniques and counterstained with hematoxylin (blue). Total cells and positive cells were counted under the microscope. Arrows indicate intima and media ( $\mathbf{A}$ and $\mathbf{C}$ ) or examples of positive cells (D, red). (A) The aortic wall. (B) A heart section. (C) The aortic root region. A-C were labeled with Sca-1 antibody and photographed at lower magnification. (D) Sections from aortic root regions at a higher magnification. (E) The graph shows data of means ( \pm SEM) from eight animals per group.

like and round-shaped cells. Over $60 \%$ of cells displayed a monolayer structure consisting of fibroblast-like cells, while $20 \%$ had a circular structure surrounded by a monolayer of cells (Figure 2, A-D). Some cells formed islands appearing as round-shaped cells. In some areas, adipocyte-like cells, dividing cells, and cells with large nuclei were observed (Figure 2, E and F). In contrast, cultured cells from the intima and media of the artery displayed an SMC morphology, i.e., a hill-and-valley pattern (Figure 2, G and H). These cells were negative for Sca-1, c-kit, CD34, and Flk1 as identified by immunostaining (data not shown).

Isolation and differentiation of Sca- $1^{+}$cells. To characterize adventitial progenitor cells, Sca- $1^{+}$cells were isolated from primary cultures with microbeads and with FACS. More than $90 \%$ of microbead-isolated cells appeared Sca- $1^{+}$(Figure $3 \mathrm{~A}$ ) and Lin $^{-}$(Figure 3B). Interestingly, a large proportion of Sca- $1^{+}$cells were stained with anti-c-kit antibodies, i.e., Sca- $1^{+} \mathrm{c}-\mathrm{kit}^{+} \mathrm{Lin}^{-}$(Figure 3, B-F), indicating characteristics of progenitor cells. In order to confirm the results obtained with microbeads, we also isolated Sca- $1^{+}$cells from the primary cultures using FACS. About $10 \% \pm 5 \%$ of total cultured cells expressed Sca- 1 marker; these cells were sorted and stained with immunofluorescence. As shown in Figure 4A, isolated cells displayed a single peak. A similar result of immunostaining for Sca- $1^{+} \mathrm{c}-\mathrm{kit}^{+}$cells was observed (Figure 4, B-D), implying a comparable result using both techniques for isolation of Sca- $1^{+}$cells.

If these isolated Sca- $1^{+}$cells were stem cells, it would be possible to stimulate the cells to differentiate into other types of cells, e.g., SMCs and endothelial cells. Several methods were used to verify cell differentiation. First, mRNA levels of the SMC markers smooth muscle actin, SM22, calponin, and smooth muscle myosin heavy chain were absent or very low in Sca- $1^{+}$cells when the cells were cultured in stem cell culture medium, but these markers were strongly expressed when the cells were stimulated with PDGF-BB for 3 days (Figure 5A). Second, SM22 promoter-driven LacZ gene expression was strictly expressed in vascular SMCs in smooth muscle-LacZ (SM-LacZ) mice. When Sca- $1^{+}$cells were isolated from the cultured adventitial cells of SM-LacZ mice and stimulated with PDGF-BB, the majority of cells were positive for $\beta$-gal staining (Figure 5B). As a control, Sca-1- cells did not show $\beta$-gal staining. These results suggest that $\mathrm{Sca}-1^{+}$cells can differentiate into SMCs and that the purity of Sca- $1^{+}$cells is high. Finally, we also stained isolated cells with specific antibodies against SMC markers. Immunostaining for $\alpha$-actin, calponin, and smooth mus- 

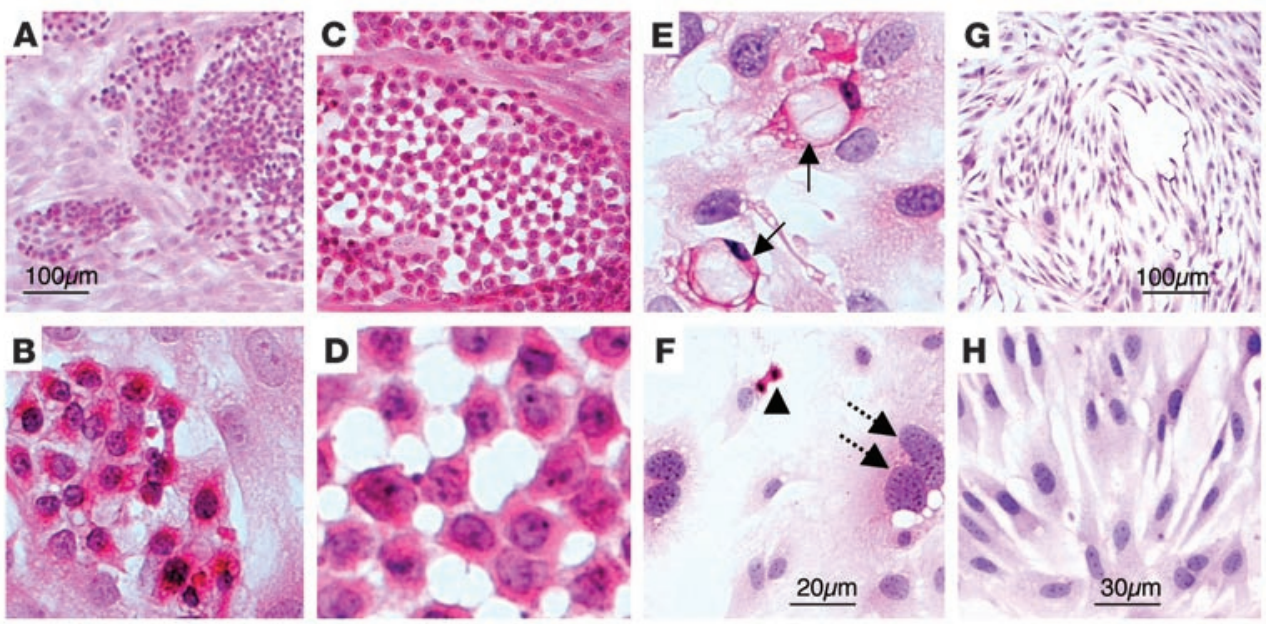

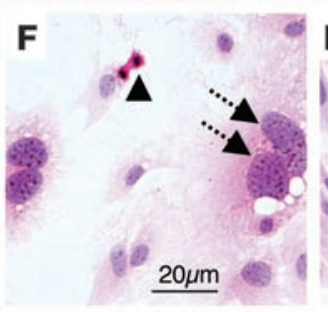

\section{Figure 2}

H\&E staining of cultured adventitial and medial cells. Adventitial $(\mathbf{A}-\mathbf{F})$ and medial $(\mathbf{G}$ and $\mathbf{H})$ tissues from aortic roots were explanted onto a slide chamber bottle and cultivated in the medium for culturing stem cells for 5 days. Cells were fixed with $4 \%$ formalin and stained with H\&E. Note adipocyte-like cells (arrows), a dividing cell (arrowhead), and large nuclei (arrows with dotted lines). cle myosin heavy chain confirmed that $\mathrm{Sca}-1^{+}$cells were positive after PDGF-BB stimulation (Figure 5C).

Non-bone marrow origin of adventitial progenitor cells. Recent reports demonstrated that bone marrow progenitor cells can fuse with other types of cells in vivo and in vitro (24-27). It is important to determine whether adventitial progenitor cells are derived from bone marrow and temporarily localized in the adventitia. Because all cells from ROSA26 mice express the LacZ gene, cells become blue after development with X-gal. Using methods established in our laboratory $(28,29)$, a chimeric C57BL/6J mouse with ROSA26 bone marrow expressing the LacZ gene was created. Six months after bone marrow reconstruction, a series of sections from aortic roots were collected, stained with X-gal, and counterstained with fast red. Occasionally a $\beta$-gal ${ }^{+}$blue cell was found (Figure $6 \mathrm{~A}$ ), and these were identified as mononuclear cells $\left(\mathrm{CD}_{4} 5^{+}\right)$, while no $\beta$-gal ${ }^{+}$ cells were observed in sections of chimeric mice that received wildtype bone marrow (Figure 6B). Furthermore, adventitial cells cultured from chimeric mice that received ROSA2 6 bone marrow did not show any $\beta$-gal staining (Figure 6C), whereas some adventitial cells of SM-LacZ chimeric mice that received wild-type bone marrow were $\beta$-gal ${ }^{+}$in response to PDGF-BB stimulation (Figure 6D). In addition, LacZ gene expression was not detectable by RT-PCR in adventitial cells cultured from chimeric mice that received ROSA26 bone marrow (data not shown). Taken together, these results indicate that progenitor cells in the adventitia are not derived from bone marrow cells.

Sca- $1^{+}$cells differentiate into SMCs in vivo. Because Sca- $1^{+}$cells can differentiate into SMCs in vitro, we sought to determine whether these cells also differentiate into SMCs in vivo. Sca- $1^{+}$cells derived from SM-LacZ mice were $\beta$-gal ${ }^{-}$and became $\beta$-gal ${ }^{+}$after stimulation with PDGF-BB in vitro (Figure $5 \mathrm{~B}$ ). When these $\mathrm{Sca}-1^{+} \beta$-gal ${ }^{-}$cells carrying the SM-LacZ gene were transferred to the adventitial side of vein grafts in mice, they could be traced in vivo. To prevent cell proliferation in the vessel wall, the vena cava from $\mathrm{ApoE}^{-/-}$mice was irradiated, then isografted into the carotid arteries of littermates. After irradiation of the vein segments, no cell proliferation in the vessel wall was found during 7 days of culture (data not shown). When ROSA26 vein segments, which express the LacZ gene in all cells, were irradiated and grafted into wild-type mice, no $\beta$-gal ${ }^{+}$cells were found within the neointimal lesions at 4 or 8 weeks after grafting (data not shown), which indicated that irradiation prevented cell proliferation in the vessel wall. Irradiated vein segments were isografted into ApoE ${ }^{-/-}$mice, and Sca- $1^{+}$cells carrying the SM-LacZ gene were applied to the adventitia of the vein segment to envelop the vein graft. Several $\beta$-gal ${ }^{+}$cells were found in neointimal lesions, and many positive cells were found in the adventitia of vein grafts 2 weeks after grafting (Figure 7B), while no $\beta$-gal staining was present in the sections of vein grafts without cell application (Figure 7A). Interestingly, in vein grafts that received Sca- $1^{+}$cells, a large number of neointimal cells were $\beta$-gal ${ }^{+} 4$ weeks after grafting (Figure $7, \mathrm{C}$ and D). These results indicate that $\mathrm{Sca}-1^{+}$cells can differentiate into SMCs in vivo.

Sca- $1^{+}$cells participating in atherosclerosis. To semiquantify Sca- $1^{+}$ cells that can participate in the formation of atherosclerotic lesions in vivo, Sca-1+ cells from ROSA26/ApoE-/- and $\mathrm{ApoE}^{-/-}$mice were isolated, cultured, and then stained. More than $90 \%$ of these isolated cells appeared Sca- $1^{+}$. Data shown in Figure 8A indicate that $\mathrm{Sca}-1^{+}$cells from ROSA26/ApoE-/- mice were $\beta$-gal ${ }^{+}$, but that $\mathrm{Sca}-1^{+}$cells from $A$ poE $^{-/-}$mice were $\beta$-gal- (Figure $8 \mathrm{~B}$ ). When these cells carrying the LacZ gene were transferred to the adventitial side of vein grafts in mice, they could be traced in vivo. Irradiated vein segments were isografted into ApoE ${ }^{-/-}$mice, and Sca- $1^{+}$cells were
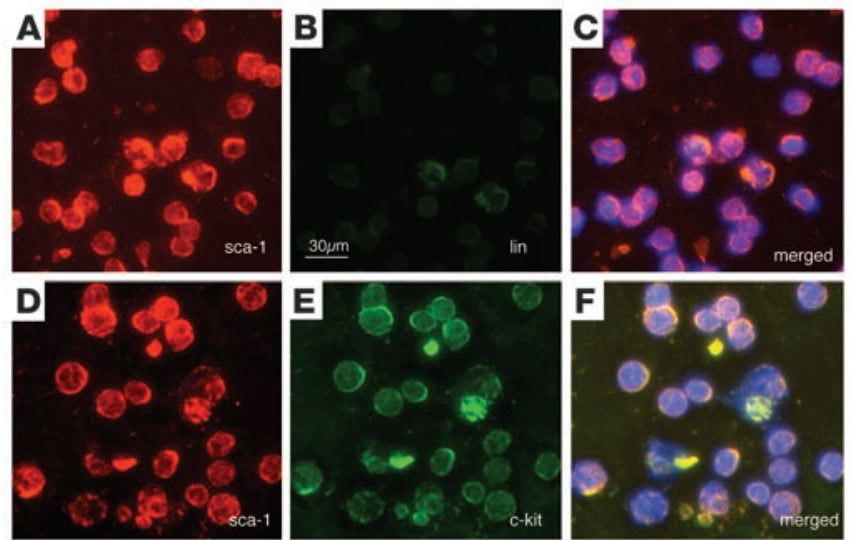

\section{Figure 3}

Immunostaining for Sca- $1^{+}$cells isolated with microbeads. Primary cultured adventitial cells were isolated with microbeads coupled with antiSca-1 antibody. The isolated cells were smeared, double immunostained for Sca-1 and Lin (A-C) or Sca-1 and c-kit (D-F), and counterstained for nuclei. Note that more than $90 \%$ of cells are Sca-1+Lin-c-kit+ . 

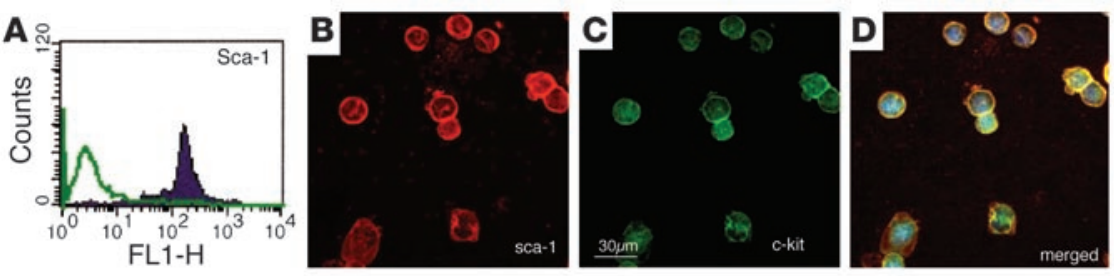

Figure 4

Immunostaining for Sca-1+ cells isolated with FACS sorting. Primary cultured adventitial cells were labeled with anti-Sca-1 antibody. Positive stained cells were sorted by FACS. (A) A single peak of isolated Sca-1+ cells (filled graph), showing no overlap with a negative control with only secondary antibody (green). (B-D) Microscopic photographs showing that the isolated cells were smeared, immunostained, and counterstained for nuclei. Note a result similar to that shown in Figure 3.

applied to the adventitia of the vein segment to envelop the vein graft. A few $\beta$-gal ${ }^{+}$cells were found in the adventitia of vein grafts 4 weeks after grafting (Figure 8C). Interestingly, about one-third of all neointimal cells were $\beta$-gal ${ }^{+}$in vein grafts that received Sca- $1^{+}$ cells (Figure 8D). Furthermore, the majority of $\beta$-gal ${ }^{+}$cells were found in the adventitial regions of vein grafts when Sca- $1^{-}$cells were transferred onto the adventitial side of the grafts (Figure 8, $\mathrm{E}$ and $\mathrm{F}$ ), which served as a control. Figure $8 \mathrm{G}$ summarizes the results from three independent experiments. About $21 \%$ of total cells were $\beta$-gal ${ }^{+}$in lesions that received Sca- $1^{+}$cells, which was significantly higher than in the group treated with Sca- $1^{-}$cells. Since about $30 \%$ of total cells in lesions are SMCs, as identified previously (23), Sca- $1^{+}$cells constituted the majority of SMCs within the lesions ( $21 \%$ of total cells). Therefore, Sca- $1^{+}$cells actively participate in the development of atherosclerosis.

\section{Discussion}

Several studies of animal models suggested that the vascular adventitia may be important in the development of atherosclerosis (16-19) and that both vascular and blood progenitor cells may contribute to the vascular diseases (2-8). The present study has provided the first evidence that abundant progenitor cells in the adventitia can differentiate into SMCs that participate in lesion formation in vein grafts. These findings could be a significant development toward understanding the potential effects of the remaining progenitor cells on vascular pathophysiology, indicating a new role for adventitial cells in the pathogenesis of atherosclerosis. For instance, Sca- $1^{+}$mesenchymal progenitor cells in the adventitia could be a source of SMCs in the media and neointima and might contribute to circulating smooth muscle progenitor cells. Furthermore, ex vivo expansion of these progenitor cells

\section{Figure 5}

Sca- $1^{+}$cells express SMC markers in response to PDGF-BB. Sca-1+ cells were isolated with microbeads coupled with anti-Sca-1 antibodies and cultured in stem cell medium without PDGF-BB or in DMEM supplemented for 3 days with 10\% FCS in the presence of PDGF-BB (10 ng/ml). (A) RNA was isolated, and RT-PCR was performed using the primers for SMC markers. (B) Adventitial Sca-1+ cells cultivated from SM-LacZ mice were cultured for 3 days with or without PDGFBB, and developed with X-gal. (C) For immunofluorescent staining, cells were labeled with antibodies against $\alpha$-actin (red), calponin (green), and smooth muscle myosin heavy chain (red) and counterstained with Hoechst 33258 (blue). SM-MHC, smooth muscle myosin heavy chain.

may hold therapeutic possibilities for cell, gene, and tissue engineering approaches to the correction of vascular disease.

One of the most important findings of this study is the abundant presence of progenitor cells in the adventitia of the aortic root compared with other territories of vessels. Fewer progenitor cells were found in the cultured cells from the adventitia of thoracic aortae compared with the root regions, and the majority of cells were Sca-1- fibroblasts (data not shown). Where do adventitial progenitor cells in the aortic root originate? Is it possible that progenitor cells from bone marrow are released into the circulation, where they become or constitute adventitial progenitor cells? Our data suggest that this was not the case, since no $\beta$-gal ${ }^{+}$cells in the adventitia were SMCs in chimeric mice expressing the LacZ gene only in bone marrow cells, and since no $\beta$-gal ${ }^{+}$ cells were found in cultured cells from the adventitia of chimeric mice carrying SM-LacZ genes in bone marrow cells (Figure 6). Furthermore, we carefully examined all sections with high magnifica-

A

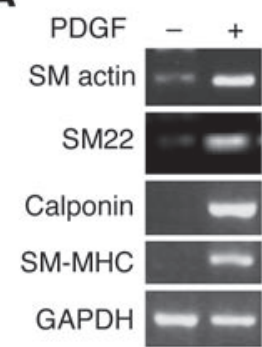

C
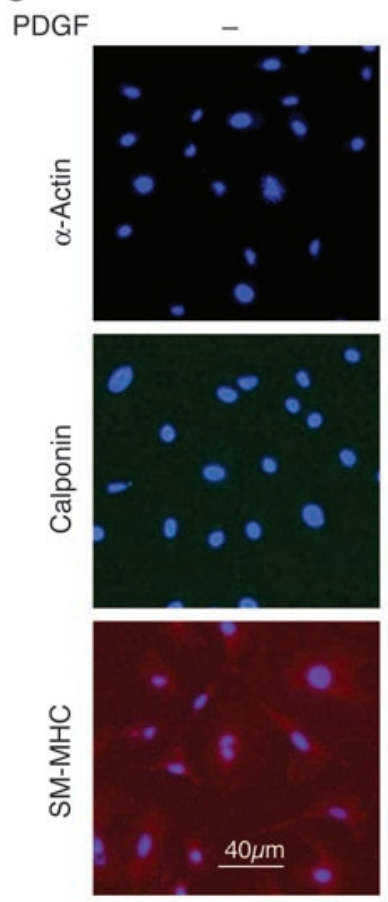

PDG
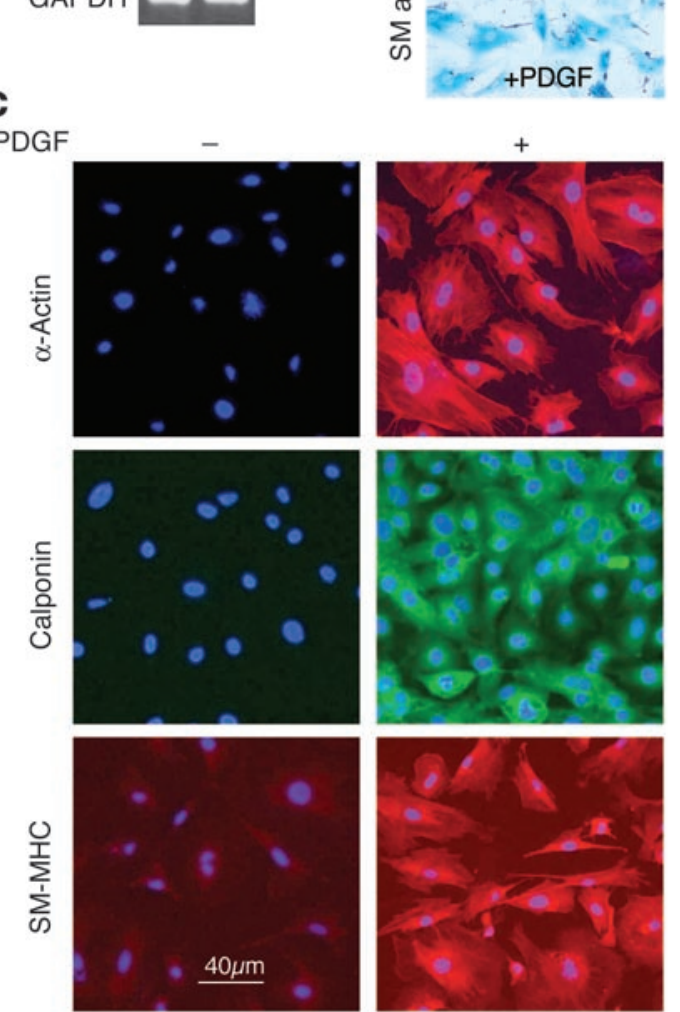

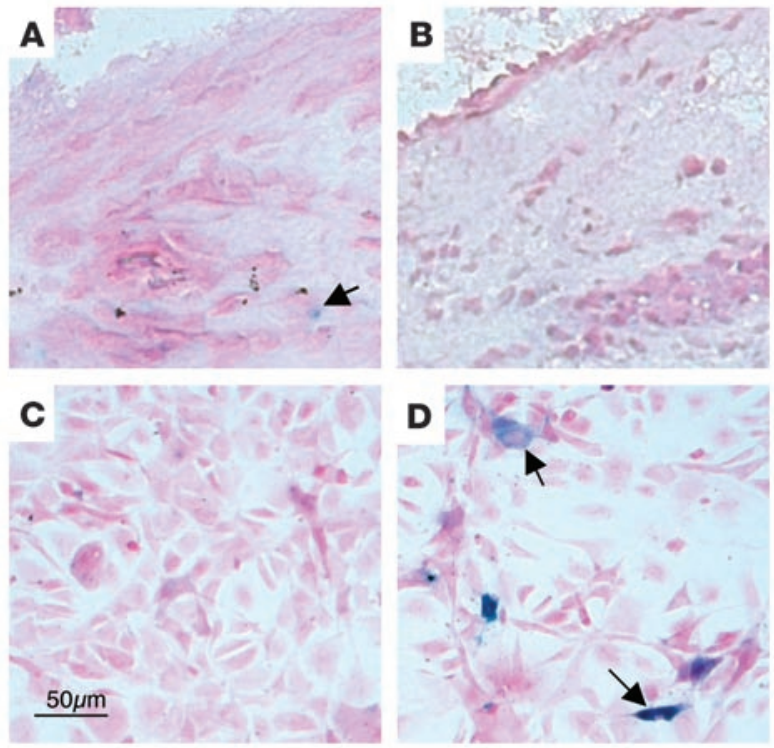

\section{Figure 6}

Creation of chimeric mice and culture of adventitial cells. Femurs of chimeric mice with ROSA26 or wild-type bone marrow were harvested and transplanted. Sections of the aortic root from chimeric mice with ROSA26 (A) or wild-type (B) bone marrow were stained with X-gal. (C and D) Cultured adventitial cells from chimeric mice with ROSA26 bone marrow $(\mathbf{C})$ or SM-LacZ mice with wild-type bone marrow (D). Cells were stimulated with PDGF-BB $(10 \mathrm{ng} / \mathrm{ml})$ for 3 days and stained with $\beta$-gal. Arrows indicate $\beta$-gal ${ }^{+}$cells.

tion $(\times 1,000)$ and did not find the cell fusion phenomenon using criteria established by other authors (25-27). This indicates that adventitial progenitor cells are not derived from bone marrow by cell fusion or retardation.

Another possibility to explain the presence of abundant progenitor cells in the adventitia is that these cells are reserved during development from embryo to adult. In the developing coronary arteries at the embryonic stage, a so-called mesenchymal cell, originating from the proepicardium throughout an epithelial-tomesenchymal transition, is the progenitor of both medial SMCs and adventitial cells (30). An extension of this model would imply that all vascular progenitors, namely the local mesenchyme and the distal mesenchyme from the proepicardium and the cardiac neural crest cells, are able to differentiate into all types of vascular cells. It is likely that adventitial cells in the root region of the aorta, reminiscent of their embryological origin, not only contribute to the formation of the tunica adventitia but also serve as a reservoir for vascular progenitor cells.

Significant controversy remains over the relative contribution of adventitial fibroblasts in neointimal growth in carotid arteries. $\mathrm{Li}$ et al. (31) showed that in the rat arterial injury model, exogenously modified and seeded carotid adventitial fibroblasts migrate in response to a factor released by SMCs. In contrast, a recent report by de Leon et al. (32) suggested that resident fibroblasts do not migrate from the adventitia in the rat carotid artery injury model. We observed that Sca- $1^{+}$mesenchymal progenitor cells had a greater ability to migrate from the adventitia to the intima, whereas in later passage of $\left(\mathrm{Sca}^{-}{ }^{-}\right)$fibroblasts cultured in DMEM medium, this was reduced. Thus, differences observed by others in the contribution of adventitial fibroblasts to neointimal formation were likely to reflect different populations or stages of differentiation.

We previously found that about $60 \%$ of SMCs in atherosclerotic lesions of vein grafts were derived from the vessel wall and the rest from recipients, possibly from circulating blood $(8,13)$. Because of suddenly elevated stretch stress to the vein graft, the majority of endothelial cells and SMCs in the vessel wall underwent apoptosis or necrosis $(11,33-35)$, suggesting a role for the remaining adventitial cells in the neointimal formation. Direct application of Sca- $1^{+}$cells to untreated vein grafts resulted in a lower number of transferred cells migrating to the neointima (data not shown). However, after treatment with irradiation, cells in vein grafts lost the ability to migrate and proliferate, leading to accumulation of transferred Sca- $1^{+}$cells in the neointimal lesion. Taken together, these findings support the hypothesis that Sca- $1^{+}$mesenchymal progenitor cells in the adventitia contribute to the accumulation of SMCs in atherosclerotic lesions via direct migration across the media and possibly via circulating blood as well.

It has been believed for a considerable time that the phenotype of SMCs within atherosclerotic lesions differs from that in the medial cells, i.e., contractive and secretory SMCs, and this is considered to be essential to the migration and proliferation of SMCs in the pathogenesis of atherosclerosis (36-38). The finding of progenitor cells from the adventitia and blood contributing to the formation of SMCs in lesions challenged this hypothesis. It means that, in atherosclerotic lesions, SMCs that display a phenotype different from that of medial cells may do so because of their origins, i.e., adventitial progenitor cells.
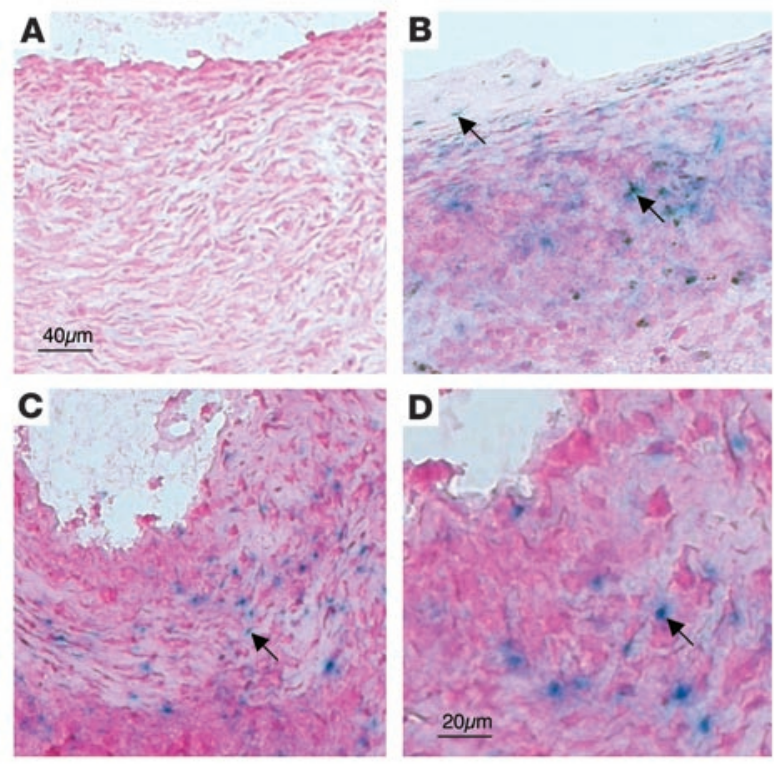

\section{Figure 7}

Sca-1+/SM-LacZ cell transfer to vein grafts in mice. Sca-1+ cells were cultivated from the adventitia of SM-LacZ/ApoE ${ }^{-/-}$mice as described for Figure 5B. Irradiated vena cava of the mouse was removed and grafted into carotid arteries of $\mathrm{ApoE}{ }^{-/-}$mice. Sca-1+/SM-LacZ cells (B-D) $\left(10^{5}\right)$ were applied onto the adventitia to envelop the vein grafts. Animals were sacrificed 2 weeks $(\mathbf{B})$ or 4 weeks (A, C, and D) after surgery, and the grafted tissue fragments were sectioned and stained with X-gal. A shows a negative control without Sca-1+ cells. Arrows indicate $\beta$-gal ${ }^{+}$cells. 


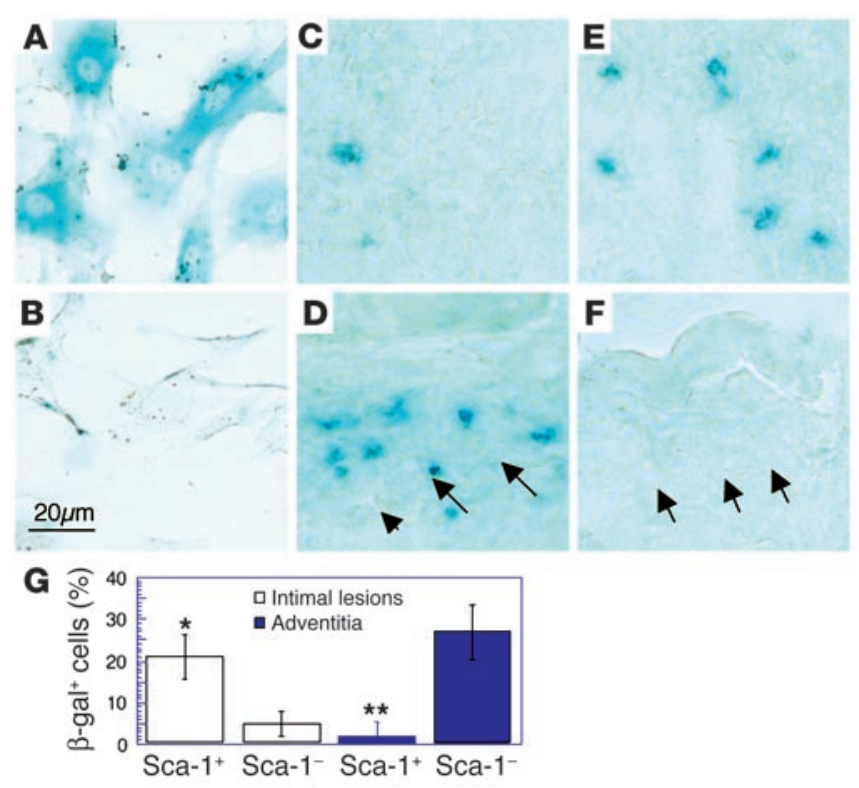

Figure 8

Sca-1+/ROSA26 cell transfer to vein grafts in mice. Sca-1+ cells cul-

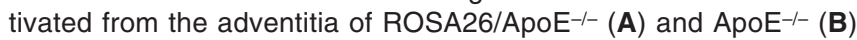
mice were stained with X-gal. Irradiated vena cava of the mouse was removed and grafted into carotid arteries of $\mathrm{ApoE}^{-/-}$mice. Sca-1+ (C, D, and E) or Sca-1- (F) cells $\left(1^{5}\right)$ were applied onto the adventitia to envelop the vein grafts. Animals were sacrificed 4 weeks after surgery, and the grafted tissue fragments were sectioned and stained with X-gal. For cell counting, Hoechst counterstain was used. C and $\mathbf{E}$ represent the adventitial area of the grafted tissue. $\mathbf{D}$ and $\mathbf{F}$ focus on the neointimal and medial layers. Note $\beta$-gal ${ }^{+}$cells, stained blue. Arrows indicate the border of the neointima and media. $(\mathbf{G})$ The graphic data are means ( \pm SEM) of five animals per group. *Significant difference in intimal lesions between Sca- $1^{+}$and Sca-1- group, $P<0.001$. ${ }^{*}$ Significant difference in the adventitia between Sca-1 ${ }^{+}$and Sca-1- group, $P<0.001$.

On the other hand, our data cannot rule out the mechanism of cell fusions between progenitor and existing SMCs in the intima during the formation of neointimal lesions as shown in Figure 7. In other words, there is a possibility that $\mathrm{Sca}-1^{+}$cells applied to the adventitia migrate into the intima, where they fuse with SMCs to form "neo-SMCs," which might have a higher ability to proliferate. In fact, researchers in the SMC field have discovered that a fair number of fully differentiated SMCs in the vessel are tetraploid (39, 40 ), which is related to a higher ability of proliferation (41). Fusion itself may also be a naturally occurring mechanism during injury or atherogenesis. Therefore, further investigations should focus on clarification of whether neo-SMCs are completely derived from progenitor cells, a fusion product of two types of cells, or both.

Accumulating evidence indicates the existence of two types of circulating endothelial cells. About $5 \%$ of these circulating cells are bone marrow-derived endothelial progenitor cells originated from hematopoietic stem cells $(42,43)$, which are positive for CD34 and Flk1 or VEGFR2 $(44,45)$. The major proportion $(>95 \%)$ of endothelial (progenitor) cells in blood originates from non-bone marrow tissues $(42,46)$. Yamashita et al. (47) reported that Flk1 ${ }^{+}$ embryonic stem cells were capable of differentiating into both endothelial cells and SMCs in vitro and in vivo in response to VEGF or PDGF-BB. Recently, we demonstrated that endothelial cells in vein grafts were completely lost during the first 4 weeks and replaced by progenitor cells (29). In the present study, we found that Sca- $1^{+}$cells cultivated from the adventitia can also differentiate in vitro into endothelial cells that show CD34, CD31, and VE-cadherin staining in response to VEGF stimulation (data not shown). These findings suggest that adventitial Sca- $1^{+}$cells also have the potential to differentiate into both endothelial cells and SMCs, which might be released into the blood to form circulating progenitor cells. To verify this hypothesis, however, further studies would be needed.

\section{Methods}

Mice. All animal experiments were performed according to protocols approved by the Institutional Committee for Use and Care of Laboratory Animals. ApoE-deficient and ROSA26 (48) mice were purchased from The Jackson Laboratory (Bar Harbor, Maine, USA). The ROSA2 6 mouse is a $\beta$-gal transgenic mouse produced by random retroviral LacZ gene insertion into embryonic stem cells and expresses $\beta$-gal activity in adult tissues (48). The $\beta$-gal activity of cells from both mice is mainly localized in the nucleus. $\mathrm{ApoE}^{-/-}$mice were crossed with ROSA26 mice in our laboratory, and heterozygous offspring were mated to produce ApoE-deficient mice expressing $\beta$-gal in all cells (ROSA26/ApoE-/-). Transgenic SM-LacZ mice expressing $\beta$-gal under the control of the smooth muscle-specific protein SM22 promoter have been described (49). ApoE $/ /-$ mice were crossed with $\mathrm{SM}$-LacZ mice, and heterozygous offspring were mated to produce ApoE-deficient mice expressing $\beta$-gal in arterial SMCs as described previously $(8,50)$. ROSA26/ApoE-/-, SM-LacZ, and SM-LacZ/ApoE-/mice were bred in our laboratory.

Three genotypes of $\mathrm{LacZ}^{-/-}, \mathrm{LacZ}^{+/-}$, and $\mathrm{LacZ}^{+/+}$mice were identified using The Jackson Laboratory's PCR protocol (primers: 5'-ATCCTCTGCATGGTCAGGTC-3' and 5'-CGTGGCCTGATTCATTCC-3'). For ApoE ${ }^{-/-}$mouse genotyping, a similar protocol was used with primers oIMR180, 5'-GCCTAGCCGAGGGAG AGCCG-3'; oIMR181, 5'-TGTGACTTGGGAGCTCTGCAGC-3'; and oIMR182, 5'-GCCGCCCCGACTGCATCT- $3^{\prime}$. The mice were maintained on a $12: 12$-hour light/dark cycle at $22^{\circ} \mathrm{C}$ and received food and water ad libitum. All mice were of a C57BL/ 6 genetic constitution and were bred in our laboratory.

Immunobistochemical staining. The procedure used in the present study was similar to that described previously (51). Briefly, serial 5 - $\mu \mathrm{m}$-thick frozen sections were overlaid with rat mAb's against mouse SSEA-1 (Chemicon Europe Ltd., Hampshire, United Kingdom), Sca-1, c-kit, CD31, CD34, Flk1, CD133 (Abcam Ltd., Cambridge, United Kingdom), MAC-1 (CD11b/18) (Biosciences Pharmingen, San Diego, California, USA), and $\alpha$-actin (SigmaAldrich, St. Louis, Missouri, USA). Sections were incubated with bridging rabbit anti-rat antibody and alkaline phosphatase antialkaline phosphatase (APAAP) complex (DakoCytomation, Glostrup, Denmark) and developed using a substrate solution (SigmaAldrich). The sections were counterstained with hematoxylin. Semiquantitative evaluation was performed at $\times 400$ magnification. The number of positive stained cells in the vessel wall was counted and expressed as the percentage of total nuclei.

Adventitial cell culture and Sca-1 $1^{+}$cell isolation. The aortic arch and root together with a proportion of the heart from ROSA26/ApoE-/-, $\mathrm{ApoE}^{-/-}$, and SM-LacZ/ApoE-/- mice were removed. Under a dissection microscope, we carefully harvested adventitial tissues by cutting off the heart tissue and removing the aortic media and intima. Under the microscope, it is relatively easy to distinguish the adventitia and heart and aortic media. The heart and aortic media are condensed tissues, from which the adventitial tissues were dissected. The adventitial 
tissues were cut into pieces (about $0.5 \mathrm{~mm}$ ) and explanted onto a slide chamber bottle. The bottle was inverted in a $\mathrm{CO}_{2}$ incubator at $37^{\circ} \mathrm{C}$ for 3 hours. The bottle was turned up, and stem cell medium (American Type Culture Collection, Rockville, Massachusetts, USA) containing leukemia inhibitory factor $(10 \mathrm{ng} / \mathrm{ml})$ and 2-mercaptoethanol $(0.1$ $\mathrm{mM}$ ) was added and incubated for 5-7 days. Medium was changed every 2 days. As a control, aortic intima and media, and heart tissues were also cultivated using the same procedure as described for the adventitial cell culture. Cell morphology and immunostaining were compared between adventitial and intima/media cells.

To isolate Sca- $1^{+}$cells, the primary cultured cells were applied to microbeads according to the manufacturer's instructions. Briefly, cells were dispersed with trypsin and washed with PBS containing $0.5 \%$ BSA, and incubated with anti-Sca-1 immunomagnetic microbeads (Miltenyi Biotec GmbH, Bergisch Gladbach, Germany). The cell suspension was added to a column equipped with a magnetic cell sorting system (MACS). After washing, Sca- $1^{+}$cells were collected. For higher purity of Sca- $1^{+}$cells, a second column was used to repeat the process. Purity and viability of isolated Sca- $1^{+}$ cells were evaluated by immunostaining and trypan blue exclusion, respectively. Isolated cells $\left(10^{3}\right)$ in stem cell medium were seeded in a slide bottle and incubated at $37^{\circ} \mathrm{C}$ for 3 days. After trypsinEDTA treatment, $5 \times 10^{4}$ cells were seeded in a 100 - $\mathrm{ml}$ culture flask and incubated for 3 days. These cells were used for in vitro and in vivo studies. For in vitro cell differentiation, $\mathrm{Sca}-1^{+}$cells were cultivated with DMEM supplemented with $15 \%$ FCS and $10 \mathrm{ng} / \mathrm{ml}$ mouse PDGF-BB (Sigma-Aldrich) for 3 days.

FACS analysis. The procedure used for cell sorting was similar to one described elsewhere (52). In short, primary cultured cells in suspension were labeled with FITC-conjugated rat anti-mouse Sca-1 antibody (Abcam Ltd.) and sorted with a FACSVantage SE flow cytometer (Becton Dickinson Immunocytometry Systems, Mountain View, California, USA). Forward and $90^{\circ}$ side scatter were used to identify and gate positive and negative fractions. An unrelated antibody was used for background subtraction for each sample. Purity of sorted cells was determined by FACS and microscope analysis.

Immunofluorescent staining. The procedure used for immunofluorescent staining was similar to that described previously (35). Briefly, cell smears, frozen sections, and cultured cells were labeled with rat $\mathrm{mAb}$ 's against Sca-1, hematopoietic lineages (Lin, including CD34, CD45, CD20, CD45RO, CD8, and TER-199), c-kit, CD31, CD34 (Abcam Ltd.), and MAC-1 (CD11a/18b), or a rabbit anti-vWF antibody (PharMingen), and visualized with swine antirabbit Ig or rabbit anti-rat Ig conjugated with FITC (Dakopatts) or Cy3. For double staining, biotin-labeled Ig was used.

$R T$-PCR. The procedure used for RT-PCR was similar to one described elsewhere (47). Total RNA was prepared with an RT-PCR Miniprep Kit (CLONTECH Laboratories Inc., Palo Alto, California, USA). The following primers were used: SM22, 5'-GCAGTCCAAAATTGAGAAGA$3^{\prime}$ and $5^{\prime}$-CTGTTGCTGCCCATTTGAAG-3'; $\alpha$-actin, $5^{\prime}$-ACGGCCGCCTCCTCTTCCTC-3' and 5'-GCCCAGCTTCGTCGTATTCC-3'; smooth muscle myosin heavy chain, 5'-GACAACTCCTCTCGCTTTGG-3' and 5'-GCTCTCCAAAAGCAGGTCAC-3'; h1-calponin, 5'-GATACGAATTCAGAGGGTGCAGACGGAGGCTC-3' and 5'-GATACAAGCTTTCAATCCACTCTCTCAGCTCC-3'; GAPDH, 5'-CGGAGTCAACGGATTTGGTCGTAT-3', and 5'-AGCCTTCTCCATGGTGGTGAAGAC-3'.

Creation of chimeric mice. The procedure used for creating chimeric mice was similar to that described previously $(8,13)$. Briefly, donor mice were killed and the femurs and tibiae were removed aseptically.
Marrow cavities were flushed with Ca- and Mg-free HBSS (GIBCO BRL; Invitrogen Corp., Carlsbad, California, USA) using a 25-gauge needle attached to a syringe. Single-cell suspensions were prepared by repeat pipetting, and the cell preparations were passed through a nylon mesh to remove particulate matter. Cells were washed twice in HBSS, counted using a hemocytometer, and resuspended at $3 \times 10^{7}$ cells per milliliter before transplantation. Six- to eight-weekold mice received a lethal dose of whole-body $x$-ray irradiation $(9.5$ Gy) as described previously (8). The irradiated recipients received $1 \times 10^{7}$ bone marrow cells in $0.3 \mathrm{ml}$ RPMI 1640 by tail vein injection. Vein grafts were performed 4 weeks after bone marrow transfer. The efficiency of bone marrow transplantation was monitored by $\beta$-gal staining and $\mathrm{Y}$ chromosome in situ hybridization for bone marrow sections of chimeric mice $(8,13)$. Two types of chimeric mice

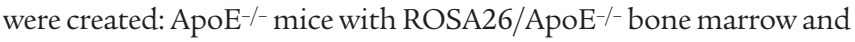
$\mathrm{SM}-\mathrm{LacZ} / \mathrm{ApoE}^{-/-}$mice with ApoE ${ }^{-/-}$bone marrow.

Vein graft and cell transfer. The vein graft procedure was similar to that described previously $(33,35)$. Briefly, 3-month-old mice were anesthetized with sodium pentobarbital $(50 \mathrm{mg} / \mathrm{kg}$ body weight intraperitoneally). The vena cava was harvested from the donor and irradiated (50 Gy). In the recipient, the right common carotid artery was mobilized free from the bifurcation at the distal end toward the proximal and cut in the middle, and a cuff was placed at the end. The cuff was made from an autoclavable nylon tube $0.63 \mathrm{~mm}$ in diameter outside and $0.5 \mathrm{~mm}$ inside (Portex Ltd., Hythe-Kent, United Kingdom). The artery was turned inside out over the cuff and ligated. The vein segment was grafted between the two ends of the carotid artery by sleeving of the ends of the vein over the artery cuff and ligating them together with an 8-0 suture. For cell transfer, Sca- $1^{+}$or Sca- $1^{-}$cells $\left(10^{5}\right.$ in $\left.50 \mu \mathrm{l}\right)$ were mixed with Matrigel $(5 \mathrm{mg} / \mathrm{ml}$; Becton Dickinson Labware, Bedford, Massachusetts, USA) at $4^{\circ} \mathrm{C}$, applied to the adventitia enveloping the graft. The wound was then closed. The complete grafting procedure required 30-40 minutes.

$X$-gal staining. Mice were anesthetized and initially perfused with $0.9 \% \mathrm{NaCl}$ solution and subsequently perfusion-fixed with $2 \%$ formaldehyde and $0.2 \%$ glutaraldehyde ( $\mathrm{pH} 7.2$ ) for 2 and 10 minutes, respectively. Vein segments were harvested, the samples were fixed with $2 \%$ formaldehyde and $0.2 \%$ glutaraldehyde at $4{ }^{\circ} \mathrm{C}$ for 24 hours, and $5-\mu \mathrm{m}$ sections were prepared. The procedure for X-gal staining was similar to that described previously (53). Briefly, sections were incubated at $37^{\circ} \mathrm{C}$ for 18 hours in PBS supplemented with $1 \mathrm{mg} / \mathrm{ml}$ $\mathrm{X}$-gal (Sigma-Aldrich), $5 \mathrm{mM}$ potassium ferricyanide, $5 \mathrm{mM}$ potassium ferrocyanide, and $2 \mathrm{mM} \mathrm{MgCl}_{2}$. Sections were rinsed with $3 \%$ DMSO in PBS and counterstained with Hoechst $33258(1 \mu \mathrm{g} / \mathrm{ml})$. Positive cells were enumerated under the microscope.

Statistical analysis. Statistical analyses were performed on a Macintosh computer using the Mann-Whitney $U$ test and ANOVA. $P<0.05$ was considered significant.

\section{Acknowledgments}

This work was supported by grants from the British Heart Foundation (PG/01/170) and the Oak Foundation.

Received for publication July 28, 2003, and accepted in revised form February 23, 2004.

Address correspondence to: Qingbo Xu, Department of Cardiac and Vascular Sciences, St George's Hospital Medical School, Cranmer Terrace, London SW17 0RE, United Kingdom. Phone: 44-208725-2817; Fax: 44-20-8725-2812; E-mail: q.xu@sghms.ac.uk. 
1. Ross, R., Glomset, J., and Harker, L. 1977. Response to injury and atherogenesis. Am. J. Pathol. 86:675-684.

2. Saiura, A., Sata, M., Hirata, Y., Nagai, R., and Makuuchi, M. 2001. Circulating smooth muscle progenitor cells contribute to atherosclerosis. Nat. Med. 7:382-383.

3. Han, C.I., Campbell, G.R., and Campbell, J.H. 2001. Circulating bone marrow cells can contribute to neointimal formation. J. Vasc. Res. 38:113-119.

4. Shimizu, K., et al. 2001. Host bone-marrow cells are a source of donor intimal smooth-muscle-like cells in murine aortic transplant arteriopathy. Nat. Med. 7:738-741.

5. Hillebrands, J.L., et al. 2001. Origin of neointimal endothelium and $\alpha$-actin-positive smooth muscle cells in transplant arteriosclerosis. J. Clin. Invest. 107:1411-1422.

6. Li, J., et al. 2001. Vascular smooth muscle cells of recipient origin mediate intimal expansion after aortic allotransplantation in mice. Am. J. Pathol. 158:1943-1947.

7. Sata, M., et al. 2002. Hematopoietic stem cells differentiate into vascular cells that participate in the pathogenesis of atherosclerosis. Nat. Med. 8:403-409.

8. Hu, Y., et al. 2002. Smooth muscle cells in transplant atherosclerotic lesions are originated from recipients, but not bone marrow progenitor cells. Circulation. 106:1834-1839.

9. Schwartz, S.M. 1999. The intima: a new soil. Circ. Res. 85:877-879.

10. Owens, G.K. 1995. Regulation of differentiation of vascular smooth muscle cells. Physiol. Rev. 75:487-517.

11. Mayr, M., et al. 2000. Biomechanical stress-induced apoptosis in vein grafts involves p38 mitogen-activated protein kinases. FASEB J 14:261-270.

12. Simper, D., Stalboerger, P.G., Panetta, C.J., Wang, S., and Caplice, N.M. 2002. Smooth muscle progenitor cells in human blood. Circulation. 106:1199-1204.

13. Hu, Y., et al. 2002. Both donor and recipient origins of smooth muscle cells in vein graft atherosclerotic lesions. Circ. Res. 91:e13-e20.

14. Sata, M., et al. 2003. Origin of smooth muscle progenitor cells: different conclusions from different models. Response. Circulation. 107:106e-107e.

15. Tanaka, K., Sata, M., Hirata, Y., and Nagai, R. 2003. Diverse contribution of bone marrow cells to neointimal hyperplasia after mechanical vascular injuries. Circ. Res. 93:783-790.

16. Shi, Y., et al. 1996. Adventitial myofibroblasts contribute to neointimal formation in injured porcine coronary arteries. Circulation. 94:1655-1664.

17. Zalewski, A., and Shi, Y. 1997. Vascular myofibroblasts. Lessons from coronary repair and remodeling. Arterioscler. Thromb. Vasc. Biol. 17:417-422.

18. Sartore, S., et al. 2001. Contribution of adventitial fibroblasts to neointima formation and vascular remodeling: from innocent bystander to active participant. Circ. Res. 89:1111-1121.

19. Rey, F.E., and Pagano, P.J. 2002. The reactive adventitia: fibroblast oxidase in vascular function.
Arterioscler. Thromb. Vasc. Biol. 22:1962-1971.

20. Wilcox, J.N., Waksman, R., King, S.B., and Scott, N.A. 1996. The role of the adventitia in the arterial response to angioplasty: the effect of intravascular radiation. Int. J. Radiat. Oncol. Biol. Phys. 36:789-796.

21. Shi, Y., et al. 1997. Remodeling of autologous saphenous vein grafts. The role of perivascular myofibroblasts. Circulation. 95:2684-2693.

22. Zou, Y., et al. 1998. Mouse model of venous bypass graft arteriosclerosis. Am. J. Pathol. 153:1301-1310.

23. Dietrich, H., et al. 2000. Rapid development of vein graft atheroma in ApoE-deficient mice. Am.J. Pathol. 157:659-669.

24. Terada, N., et al. 2002. Bone marrow cells adopt the phenotype of other cells by spontaneous cell fusion. Nature. 416:542-545.

25. Wang, X., et al. 2003. Cell fusion is the principal source of bone-marrow-derived hepatocytes. Nature. 422:897-901.

26. Vassilopoulos, G., Wang, P.R., and Russell, D.W. 2003. Transplanted bone marrow regenerates liver by cell fusion. Nature. 422:901-904.

27. Vassilopoulos, G., and Russell, D.W. 2003. Cell fusion: an alternative to stem cell plasticity and its therapeutic implications. Curr. Opin. Genet. Dev. 13:480-485.

28. Hu, Y., Davison, F., Zhang, Z.G., and Xu, Q. 2003. Endothelial replacement and angiogenesis in arteriosclerotic lesions of allografts are contributed by circulating progenitor cells. Circulation. 108:3122-3127.

29. Xu, Q., Zhang, Z., Davison, F., and Hu, Y. 2003. Circulating progenitor cells regenerate endothelium of vein graft atherosclerosis, which is diminished in apoE-deficient mice. Circ. Res. 93:e76-e86.

30. Dettman, R.W., Denetclaw, W., Jr., Ordahl, C.P., and Bristow, J. 1998. Common epicardial origin of coronary vascular smooth muscle, perivascular fibroblasts, and intermyocardial fibroblasts in the avian heart. Dev. Biol. 193:169-181.

31. Li, G., Chen, S.J., Oparil, S., Chen, Y.F., and Thompson, J.A. 2000. Direct in vivo evidence demonstrating neointimal migration of adventitial fibroblasts after balloon injury of rat carotid arteries. Circulation. 101:1362-1365.

32. De Leon, H., Ollerenshaw, J.D., Griendling, K.K., and Wilcox, J.N. 2001. Adventitial cells do not contribute to neointimal mass after balloon angioplasty of the rat common carotid artery. Circulation. 104:1591-1593.

33. Leitges, M., et al. 2001. Exacerbated vein graft arteriosclerosis in protein kinase $\mathrm{C} \delta$-null mice. J. Clin. Invest. 108:1505-1512. doi:10.1172/ JCI200112902.

34. Mayr, M., Hu, Y., Hainaut, H., and Xu, Q. 2002. Mechanical stress-induced DNA damage and racp38MAPK signal pathways mediate p53-dependent apoptosis in vascular smooth muscle cells. FASEBJ. 16:1423-1425.

35. Mayr, U., et al. 2002. Loss of p53 accelerates neointimal lesions of vein bypass grafts in mice. Circ. Res. 90:197-204.

36. Campbell, J.H., and Campbell, G.R. 1994. The role of smooth muscle cells in atherosclerosis. Curr.
Opin. Lipidol. 5:323-330.

37. Ross, R., and Glomset, J.A. 1976. The pathogenesis of atherosclerosis (first of two parts). N. Engl. J. Med. 295:369-377.

38. Ross, R., and Glomset, J.A. 1976. The pathogenesis of atherosclerosis (second of two parts). N. Engl. J. Med. 295:420-425.

39. Barrett, T.B., Sampson, P., Owens, G.K., Schwartz, S.M., and Benditt, E.P. 1983. Polyploid nuclei in human artery wall smooth muscle cells. Proc. Natl. Acad. Sci. U. S. A. 80:882-885.

40. Goldberg, I.D., et al. 1984. Isolation and culture of a tetraploid subpopulation of smooth muscle cells from the normal rat aorta. Science. 226:559-561.

41. Owens, G.K. 1989. Growth response of tetraploid smooth muscle cells to balloon embolectomyinduced vascular injury in the spontaneously hypertensive rat. Am. Rev. Respir. Dis. 140:1467-1470.

42. Lin, Y., Weisdorf, D.J., Solovey, A., and Hebbel, R.P. 2000. Origins of circulating endothelial cells and endothelial outgrowth from blood. J. Clin. Invest. 105:71-77.

43. Rafii, S. 2000. Circulating endothelial precursors: mystery, reality, and promise. J. Clin. Invest. 105:17-19.

44. Asahara, T., et al. 1997. Isolation of putative progenitor endothelial cells for angiogenesis. Science. 275:964-967.

45. Crosby, J.R., et al. 2000. Endothelial cells of hematopoietic origin make a significant contribution to adult blood vessel formation. Circ. Res. 87:728-730.

46. Gunsilius, E., et al. 2000. Evidence from a leukaemia model for maintenance of vascular endothelium by bone-marrow-derived endothelial cells. Lancet. 355:1688-1691.

47. Yamashita, J., et al. 2000. Flk1-positive cells derived from embryonic stem cells serve as vascular progenitors. Nature. 408:92-96.

48. Zambrowicz, B.P., et al. 1997. Disruption of overlapping transcripts in the ROSA beta geo 26 gene trap strain leads to widespread expression of betagalactosidase in mouse embryos and hematopoietic cells. Proc. Natl. Acad. Sci. U. S. A. 94:3789-3794.

49. Moessler, H., et al. 1996. The SM 22 promoter directs tissue-specific expression in arterial but not in venous or visceral smooth muscle cells in transgenic mice. Development. 122:2415-2425.

50. Ludewig, B., et al. 2000. Linking immune-mediated arterial inflammation and cholesterol-induced atherosclerosis in a transgenic mouse model. Proc. Natl. Acad. Sci. U. S. A. 97:12752-12757.

51. Hu, Y., Zou, Y., Dietrich, H., Wick, G., and Xu, Q. 1999. Inhibition of neointima hyperplasia of mouse vein grafts by locally applied suramin. Circulation. 100:861-868.

52. Scopes, J., et al. 2001. Correction of stromal cell defect after bone marrow transplantation in aplastic anaemia. Br. J. Haematol. 115:642-652.

53. Hu, Y., Baker, A.H., Zou, Y., Newby, A.C., and Xu, Q. 2001. Local gene transfer of tissue inhibitor of metalloproteinase- 2 influences vein graft remodeling in a mouse model. Arterioscler. Thromb. Vasc. Biol. 21:1275-1280. 